"This document is the Accepted Manuscript version of a Published Work that appeared in final form in [Organic Letters], copyright (C) American Chemical Society after peer review and technical editing by the publisher. To access the final edited and published work see

http://pubs.acs.org/doi/abs/10.1021/acs.orglett.6b00462 ,see

http://pubs.acs.org/page/policy/articlesonrequest/index.html ]."

\title{
Polystyrene or Magnetic Nanoparticles as Support in Enantioselective Organocatalysis? A Case Study in Friedel-Crafts Chemistry
}

\author{
Sara Ranjbar, ${ }^{a}$ Paola Riente, ${ }^{a}$ Carles Rodríguez-Escrich, ${ }^{a}$ Jagjit Yadav, $^{\text {a }}$ \\ Kishore Ramineni, ${ }^{a}$ Miquel A. Pericàs ${ }^{\star, a, b}$ \\ anstitute of Chemical Research of Catalonia (ICIQ), The Barcelona Institute of Science of \\ Technology, Avda. Països Catalans, 16. 43007 Tarragona (Spain) \\ bDepartment de Química Orgànica, Universitat de Barcelona. 08080 Barcelona (Spain)
}

\begin{abstract}
Heterogenized versions of the second generation MacMillan imidazolidin-4-one are described for the first time. This versatile organocatalyst has been supported onto $1 \%$ DVB Merrifield resin and $\mathrm{Fe}_{3} \mathrm{O}_{4}$ magnetic nanoparticles through copper-catalyzed alkyne-azide cycloaddition (CuAAC) reaction. The resulting catalytic materials-have been successfully applied to the asymmetric FriedelCrafts alkylation of indoles with $\alpha, \beta$-unsaturated aldehydes. While both catalytic systems can be easily recovered and admit repeated recycling, the polystyrene-based catalyst shows higher stability and provides better stereoselectivities.
\end{abstract}

In the year 2000, MacMillan and co-workers introduced iminium ion catalysis as a new activation concept in a seminal paper that opened the organocatalysis field. ${ }^{1}$ For this novel mode of activation, they developed a chiral secondary amine integrated in an imidazolidin-4-one framework (the first generation MacMillan catalyst) whose efficiency was demonstrated in a variety of asymmetric processes involving enals. ${ }^{1,2}$ Among them, the first highly enantioselective Friedel-Crafts (FG) alkylation of pyrroles with $\alpha, \beta$-unsaturated aldehydes was developed. ${ }^{2 b}$ However, when the same strategy was attempted with less electron rich heteroaromatics such as indoles, poor results were achieved. Efforts directed to the solution of this problem led to the development of the so-called second generation MacMillan catalyst, a more active and versatile imidazolidin-4-one featuring an additional stereocenter. ${ }^{3}$ This type of organocatalysts have been successfully applied to a large variety of important transformations including cycloadditions, ${ }^{4}$ hydrogenations ${ }^{5}$ and conjugate additions. ${ }^{6}$

However, some drawbacks arise in connection with its preparation when compared to the facile synthesis of the first generation imidazolidin-4-one. For instance, the synthesis of the second generation cis-imidazolidin4-one (cis-2), requires the condensation of the phenylalanine amide derivative (1) with an excess of pivalaldehyde, using iron(III) chloride as Lewis acid (Scheme 1). This transformation yields a mixture of diastereoisomers with the undesired trans-2 as the major product. Given the manifold applications of this organocatalyst and the problems associated to its preparation, the development of a modified version that could allow for its easy recovery and multiple reuse becomes highly desirable.

The recycling of organocatalysts has been tackled from different perspectives. ${ }^{7}$ Among them, the covalent immobilization onto insoluble supports furnishes an excellent platform to simplify catalyst separation from the reaction medium. In fact, the heterogenization of the first-generation MacMillan catalyst onto a variety of solid supports such as organic polymers, ${ }^{8}$ magnetic nanoparticles ${ }^{8 \mathrm{~d}}$ and mesoporous materials ${ }^{9}$ has been reported in the literature.

Scheme 1. Reported synthesis of 2 .

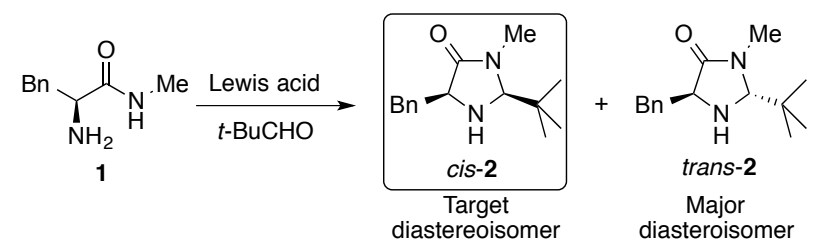


The use of copper-catalyzed alkyne-azide cycloaddition reaction (GuAAC) as a tool to anchor different organocatalysts onto polymers $^{8 \mathrm{~d}, 10}$ and magnetic nanoparticles ${ }^{8 \mathrm{~d}, 11}$ has been explored in detail with excellent results in our laboratory. We envisioned that such a late-stage immobilization would be ideal to support the versatile second-generation MacMillan catalyst (the target cis diastereomer could be previously separated). This would only require a simple modification in the starting amino acid to provide an anchoring point remote from the catalyst active site.

Herein we report on the covalent immobilization of the second generation imidazolidin-4-one organocatalyst onto 1\% DVB Merrifield resin (PS) and iron oxide magnetic nanoparticles (MNPs) according to these principles, and the use of the resulting recyclable catalysts in the enantioselective FG alkylation of indoles with $\alpha, \beta$-unsaturated aldehydes. In contrast to what one might expect, the employed support has a significant effect not only on the recyclability of the catalyst, but also on its enantioselectivity.

The monomeric species $\mathbf{5}$ was prepared as shown in Scheme 2. The sequence started with the amidation of the commercially available L-tyrosine methyl ester hydrochloride $(\mathbf{3})$. As mentioned above, the para-hydroxy substituent in the aryl group of this amino acid will be instrumental for the immobilization process. Next, condensation of the resulting amide with pivalaldehyde in presence of $\mathrm{FeCl}_{3}$ gave imidazolidin-4-one $\mathbf{4}$ as a 1:2.4 mixture of cis/trans diastereoisomers, the desired cis-4 being isolated in $17 \%$ yield after separation by column chromatography. Final propargylation led to the ready-to-anchor cis-imidazolidin-4-one $\mathbf{5}$ in $64 \%$ yield.

Scheme 2. (a) Synthesis of the second generation imidazolidin-4-one derivative 5. (b) General methodology to prepare PS-supported imidazolidinone (catalyst A) and MNPssupported imidazolidin-4-one (catalyst B).

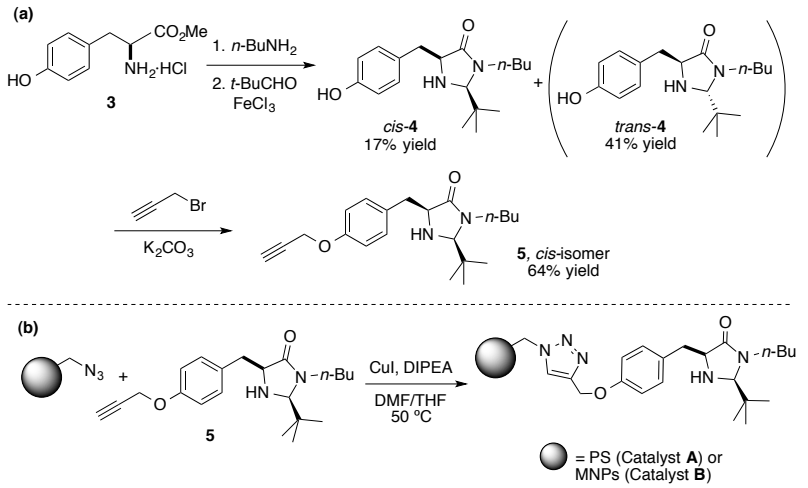

For the preparation of the PS-supported second-generation imidazolidin-4-one (catalyst $\mathbf{A}$ ) commercially available Merrifield resin ( $1 \%$ DVB, $f=0.6 \mathrm{mmol} \mathrm{g}^{-1}$ ) was converted to azidomethylpolystyrene by treatment with sodium azide, and the resulting PS-azide $\left(f=0.54 \mathrm{mmol} \mathrm{g}^{-1}\right)$ was conjugated with $\mathbf{5}$. For the preparation of catalyst $\mathbf{B}, \mathrm{Fe}_{3} \mathrm{O}_{4} \operatorname{MNPs}(5.7 \pm 1.3 \mathrm{~nm})$ prepared by thermal decomposition ${ }^{12}$ were functionalized with azide groups by ligand exchange with 3-(azidopropyltriethoxy)silane to give 3azidopropyl-MNPs $\left(f=0.72 \mathrm{mmol} \cdot \mathrm{g}^{-1}\right)$. As in the previous case, imidazolidinone $\mathbf{5}$ was immobilized using a CuAAC reaction. The functionalization of both materials was followed by infrared spectroscopy (see Supporting Information). Also, the size distribution and morphology of the MNPs were monitored by transmission electron microscopy (TEM) after each step. This allowed to rule out any agglomeration phenomena during the preparation of the immobilized catalyst (Figure 1; see Supporting Information for details).
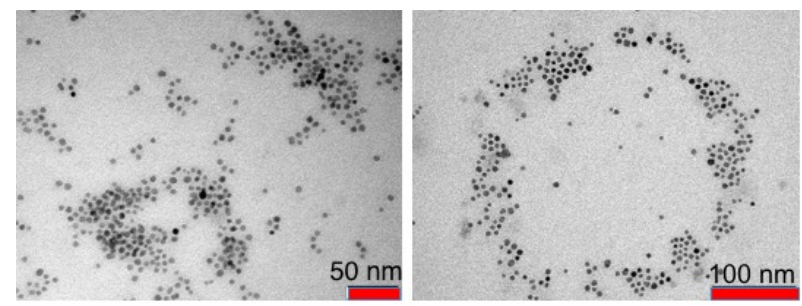

Figure 1. TEM images of MNPs before (left) and after functionalization with 5 (right). 
Next, we investigated the activity of catalysts $\mathbf{A}$ and $\mathbf{B}$ on the enantioselective FC alkylation of indoles with $\alpha, \beta$-unsaturated aldehydes. ${ }^{13}$ To this aim, the reaction between $\mathcal{N}$-methylindole $(\mathbf{6 a})$ and cinnamaldehyde (7a) was chosen to optimize the reaction conditions. The effects of solvent and temperature were studied with the polystyrene-based catalyst $\mathbf{A}$. As shown in Table 1, good results were obtained when the reaction was carried out in tetrahydrofuran, toluene or $\mathrm{CH}_{2} \mathrm{Cl}_{2}$ (entries 1, 3 and 4). While no conversion was observed in isopropyl alcohol alone (entry 2), the best result was observed in a $\mathrm{CH}_{2} \mathrm{Cl}_{2} / i$ - $\mathrm{PrOH}$ mixture at $-20{ }^{\circ} \mathrm{C}$ (entry 5). Good yields and enantioselectivities of Friedel-Crafts adduct $\mathbf{8 a}$ were also recorded with the same solvent mixture at room temperature or at $0{ }^{\circ} \mathrm{C}$ (entries 6,7$)$.

Table 1. Optimization of the Reaction Conditions for the FG Alkylation of Indoles with Enals Mediated by Catalyst $\mathrm{A}^{\mathrm{a}}$

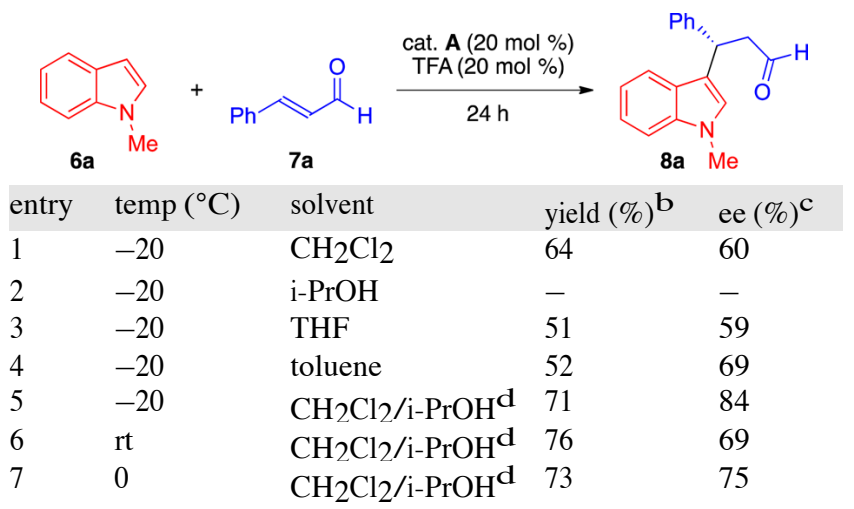

aReaction conditions: $\mathrm{N}$-methylindole $(0.3 \mathrm{mmol})$, trans-cinnamaldehyde $0.9 \mathrm{mmol})$, catalyst A $(20 \mathrm{~mol} \%)$, TFA $(0.5 \mathrm{M}, 20 \mathrm{~mol} \%)$, solvent $(1 \mathrm{~mL})$. bIsolated yield. cEnantioselectivity determined by chiral HPLC. d85:15 ratio.

The asymmetric FG reaction was next studied with representative combinations of $\alpha, \beta$-unsaturated aldehydes and indoles or $\mathcal{N}$-methylindoles under the optimized reaction conditions, using catalysts $\mathbf{A}$ and $\mathbf{B}$ (Scheme 3). To our delight, both systems displayed good tolerance toward the reaction of $\beta$-alkyl and $\beta$-aryl substituted enals $\left(Z=\operatorname{Pr}, \mathrm{Et}, \mathrm{Ph}, 4-\mathrm{ClC}_{6} \mathrm{H}_{4}, 4-\mathrm{NO}_{2} \mathrm{C}_{6} \mathrm{H}_{4}\right)$ with $\mathcal{N}$-methylindole and indole giving the corresponding adducts with good yields and enantioselectivities. On the other hand, the incorporation of a halogenated group on the $\mathrm{C}_{6}$ position of the indole moiety had a deleterious effect on the activity and enantioselectivity of catalysts $\mathbf{A}$ and $\mathbf{B}$ (products $\mathbf{8 g}$ and $\mathbf{8 h}$ ).

\section{Scheme 3. Substrate scope for Friedel-Crafts alkylation of indoles. ${ }^{a}$}

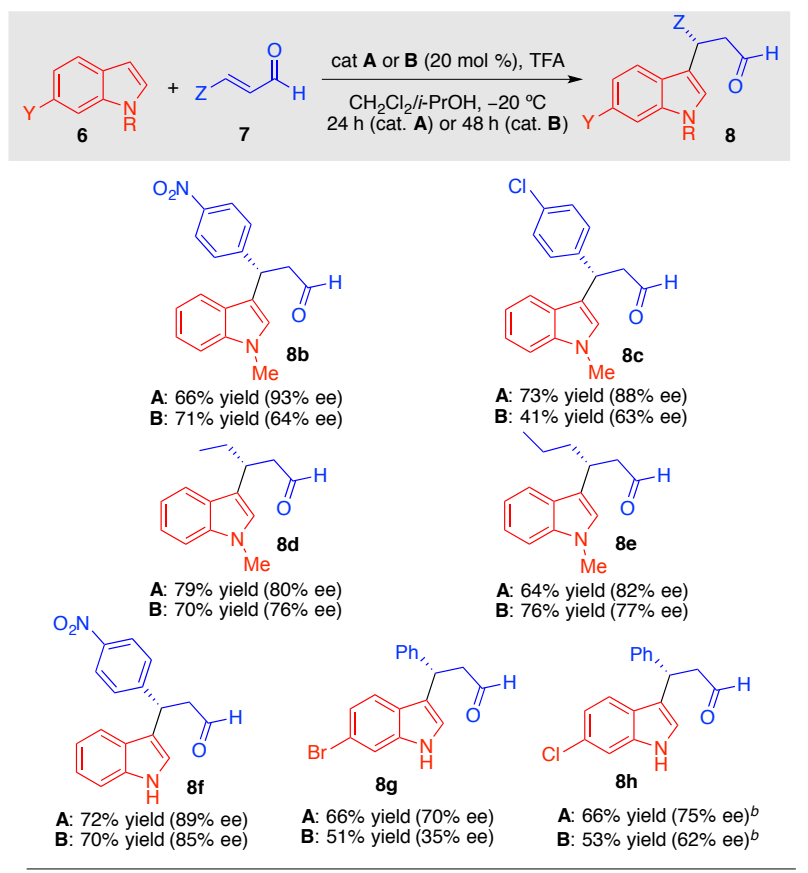




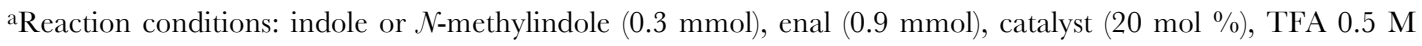
(20 mol \%), $\mathrm{CH}_{2} \mathrm{Cl}_{2} / i$ - $\mathrm{PrOH}(1 \mathrm{~mL}, 85: 15)$ at $-20{ }^{\circ} \mathrm{C}$. Isolated yields after column chromatography. Enantioselectivity determined by chiral HPLC. ${ }^{\text {b }}$ At $0{ }^{\circ} \mathrm{C}$.

The comparison seems to favour the polystyrene-based catalyst $\mathbf{A}$ in all cases. Thus, this catalyst leads to higher enantioselectivities and similar or better yields in shorter reaction times (24 hours) than the MNPsbased catalyst $\mathbf{B}$ (48 hours). A tentative explanation to this behavior can be found on the differential efficiency of inter-phase mass transfer with both types of material. Magnetic nanoparticles, in spite of its inherently high specific surface do not normally exhibit perfect dispersibility in organic solvents. ${ }^{14}$ The agglomerates formed in these conditions only allow for limited accessibility of the reagents to the catalytic sites, leading to TOFs well below its theoretical maximum. On the contrary, when polystyrene-based catalysts operate in reaction media leading to perfect swelling (as it is the case for catalyst $\mathbf{A}$ in dichloromethane), mass transfer limitations are effectively overcome so that reaction rate can approach its theoretical maximum. As far as enantioselectivity is concerned, it is well known that the surface of metal oxides nanoparticles is covered with a layer of hydroxy groups. ${ }^{15}$ A complete capping of these groups during the functionalization of the nanoparticles is virtually impossible. These residual $\mathrm{OH}$ groups might interact with the organocatalyst and/or the substrates by hydrogen bonding or by protonation, this entailing a decrease in the enantioselectivity of the process. ${ }^{16}$ In this respect, it is worth noting that no background reaction was observed using non-functionalized MNPs (alone or in the presence of TFA).

As discussed above, the most important purpose of catalyst immobilization is facilitating recycling and reuse. In the present case, the polystyrene-based catalyst $\mathbf{A}$ could be easily recovered by filtration, while catalyst $\mathbf{B}$ was separated by simple magnetic decantation. This being secured, the robustness of catalysts $\mathbf{A}$ and $\mathbf{B}$ upon reuse was studied. The reaction between trans-cinnamaldehyde and $\mathcal{N}$-methylindole was selected as a model (Table 2). For each run, equimolar amounts of TFA were added in order to recondition the catalysts.

Following the protocol detailed in the Supporting Information, catalysts $\mathbf{A}$ and $\mathbf{B}$ could be reused for five consecutive runs. A slight decline in catalytic activity was observed in both cases as the recycling progresses. As for enantioselectivity, the reactions involving the MNP-supported catalyst exhibited a slight but continuous decrease. With the PS-based catalyst $\mathbf{A}$, in turn, the ee recorded in the fifth run was still fully comparable with the initial one.

Possible explanations for the lower stability of $\mathbf{B}$ under the recycling conditions could be the occurrence of reversible agglomeration phenomena in the reaction media that could lead to a decrease of the effective surface area of the MNPs or the sensitivity toward trifluoroacetic acid of the silicon-oxygen bonds involved in catalyst immobilization that could provoke a progressive decrease in functionalization.

Table 2. Recycling and reuse experiments for catalyst $A$ and $B$.

\begin{tabular}{|c|c|c|c|c|}
\hline \multirow[b]{2}{*}{ run } & \multicolumn{2}{|l|}{$\mathbf{A}$} & \multicolumn{2}{|l|}{ B } \\
\hline & yield $(\%)$ & ee $(\%)$ & yield $(\%)$ & ee $(\%)$ \\
\hline 1 & 71 & 84 & 68 & 65 \\
\hline 2 & 70 & 86 & 65 & 54 \\
\hline 3 & 68 & 85 & 64 & 58 \\
\hline 4 & 52 & 80 & 61 & 56 \\
\hline 5 & 50 & 79 & 27 & 48 \\
\hline
\end{tabular}

In conclusion, we have developed an efficient way to support the second-generation MacMillan organocatalyst onto slightly cross-linked polystyrene (cat. A) and iron oxide magnetic nanoparticles (cat. B). The catalytic efficiency of these functional materials has been demonstrated in the enantioselective FGalkylation of indoles with $\alpha, \beta$-unsaturated aldehydes. Both catalysts could be easily recovered and reused for five consecutive runs. When the suitability of both supports is critically assessed, the polystyrene-based 
catalyst has proven to be much more active and selective than the magnetic iron oxide-based one. It seems that the polymeric nature of the support in the PS-based catalyst might offer a beneficial microenvironment to the active sites resulting in better reactivity and stereoselectivity compared to the iron oxide MNPs-based catalyst. It is suggested that magnetic nanoparticles lacking excess hydroxy functionalization on their surfaces and that do not show tendency to agglomeration, like cobalt nanoparticles coated with graphitic carbon, ${ }^{17}$ could overcome this limitation.

\section{ASSOCIATED CONTENT}

\section{Supporting Information}

Experimental details, spectroscopic data of all compounds and TEM images (PDF).

\section{AUTHOR INFORMATION}

\section{Corresponding Author}

*E-mail: mapericas@iciq.es

\section{Present Addresses}

$\dagger$ Jagjit Yadav. Polymers \& Functional Materials Division, CSIR-Indian Institute of Chemical Technology, Hyderabad, India, 500007.

$\dagger$ Kishore Ramineni. Clean Energy Research Center, Korea Institute of Science and Technology, Hwarang-ro 14gil, Seongbuk-gu, Seoul 02792.

\section{Author Contributions}

All authors have given approval to the final version of the manuscript.

Notes

The authors declare no competing financial interest.

\section{ACKNOWLEDGMENT}

This work was funded by the EU-ITN network Mag(net)icFun (PITN-GA-2012-290248) and Institute of Chemical Research of Catalonia (ICIQ) Foundation. MINECO (grant CTQ2012-38594-C02-01) and DEC Generalitat de Catalunya (Grant 2014SGR827) are gratefully acknowledged. We also thank MINECO for support through Severo Ochoa Excellence Accreditation 2014-2018 (SEV-2013- 0319).

\section{REFERENCES}

(1) Ahrendt, K. A.; Borths, C. J.; MacMillan, D. W. G. F. Am. Chem. Soc. 2000, 122, 4243.

(2) (a) Jen, W. S.; Wiener, J. J. M.; MacMillan, D. W. C. J. Am. Chem. Soc. 2000, 122, 9874. (b) Paras, N. A.; MacMillan, D. W. C. F. Am. Chem. Soc. 2001, 123, 4370.

(3) Austin, J. F.; MacMillan, D. W. C. F. Am. Chem. Soc. 2002, 124, 1172.

(4) (a) Northrup, A. B.; MacMillan, D. W. C. F. Am. Chem. Soc. 2002, 124, 2458. (b) Wilson, R. M.; Jen, W. S.; MacMillan, D. W. C. F. Am. Chem. Soc. 2005, 127, 11616.

(5) Ouellet, S. G.; Tuttle, J. B.; MacMillan, D. W. C. F. Am. Chem. Soc. 2005, 127, 32.

(6) (a) Paras, N. A.; MacMillan, D. W. C. F. Am. Chem. Soc. 2002, 124, 7894. (b) Brown, S. P.; Goodwin, N. C.; MacMillan, D. W. C. F. Am. Chem. Soc. 2003, 125, 1192.

(7) For reviews on solid-supported enantioselective organocatalysts, see: (a) Cozzi, F. Adv. Synth. Catal. 2006, 348, 1367. (b) Benaglia, M. New 7. Chem. 2006, 30, 1525. (c) Gruttadauria, M.; Giacalone, F.; Noto, R. Chem. Soc. Rev. 2008, 37 , 1666. (d) Kristensen, T. E.; Hansen, T. Eur. F. Org. Chem. 2010, 3179. (e) Mrówczyński, R.; Nan, A.; Liebscher, J. RSC Adv. 2014, 4, 5927. (f) Ferré, M.; Pleixats, R.; Man, M. W. G.; Cattöen, X. Green Chem. 2016, 18, 881. (g) Giacalone, F.; Gruttadauria, M. Chem CatChem 2016, 8,664 .

(8) Selected examples for polymer or magnetic nanoparticles-supported first generation MacMillan catalyst, see: (a) Selkälä, S. A.; Tois, J.; Pihko, P. M.; KosKinen, A. M. P. Adv. Synth. Catal. 2002, 344, 941. (b) Benaglia, M.; Celentano, G.; Cinquini, M.; Puglisi, A.; Cozzi, F. Adv. Synth. Catal. 2002, 344, 149. (c) Puglisi, A.; Benaglia, M.; Cinquini, M.; Cozzi, F.; Celentano, G. Eur. F. Org. Chem. 2004, 567. (d) Riente, P.; Yadav. J.; Pericàs, M. A. Org. Lett. 2012, 14, 3668. (e) Salvo, A. M. P.; Giacalone, F.; Noto, R.; Gruttadauria, M. ChemPlusChem 2014, 79, 857. (f) Pecchioli, T. Muthyala, M. K.; Haag, R.; Christmann, M. Beilstein f. Org. Chem. 2015, 11, 730.

(9) For mesoporous materials-supported first generation MacMillan catalyst, see: (a) Zhang, Y.; Zhao, L.; Lee, S. S.; Ying, J. Y. Adv. Synth. Catal. 2006, 348, 2027. (b) Shi, J. Y.; Wang, C. A.; Li, Z. J.; Wang, Q; Zhang, Y.; Wang, W. Chem. Eur. F. 2011, 17, 6206. (c) Puglisi, A.; Benaglia, M.; Annunziata, R.; Chiroli, V.; Porta, R.; Gervasini, A. f. Org. Chem. 2013, $78,11326$.

(10) For recent examples, see: Kasaplar, P.; Rodríguez-Escrich, C.; Pericàs, M. A. Org. Lett. 2013, 15, 3498. (b) Fan, X.; Rodríguez-Escrich, C.; Sayalero, S.; Pericàs, M. A. Chem. Eur. F. 2013, 19, 10814. (c) Henseler, A. H.; Ayats, C.; Pericàs, M. A. Adv. Synth. Catal. 2014, 356, 1795. (d) Ayats, C.; Henseler, A. H.; Dibello, E.; Pericàs, M. A. ACS Catal. 2014, 4, 3027. (e) Izquierdo, J.; Ayats, C.; Henseler, A. H.; Pericàs, M. A. Org. Biomol. Chem. 2015, 13, 4204.

(11) (a) Mak, C. A; Ranjbar, S.; Riente, P.; Rodríguez-Escrich, C.; Pericàs, M. A. Tetrahedron 2014, 70, 6169. (b) Riente, P.; Mendoza, C.; Pericàs, M. A. f. Mater. Chem. 2011, 21, 7350. 
(12) (a) Sun, S.; Zeng, H. F. Am. Chem. Soc., 2002, 124, 8204; (b) Sun, S.; Zeng, H., Robinson, D. B.; Raoux, S.; Rice, P. M.; Wang, S. X.; Li, G. F. Am. Chem. Soc. 2004, 126, 273.

(13) For selected examples of enantioselective Friedel-Crafts alkylation of indoles with $\alpha, \beta$-unsaturated aldehydes, see: (a) King, H. D.; Meng, Z.; Denhart, D.; Mattson, R.; Kimura, R.; Wu, D.; Gao, Q.; Macor, J. E. Org. Lett. 2005, 7, 3437.

(b) Shi; Z.-H; Sheng, H.; Yang, K.-F.; Jiang, J.-X; Lu, Y.; Xu, L.-W. Eur. F. Org. Chem. 2011, 66. (c) Liang, X.; Li, S.; Su, W. Tetrahedron Lett. 2012, 53, 289; for an example with an immobilized peptidic catalyst, see: (d) Akagawa, K.; Suzuki; R.; Kudo, K. Adv. Synth. Catal. 2012, 354, 1280.

(14) Kharisov, B. I.; Dias, H. V. R., Kharissova, O. V.; Vázquez, A.; Peña, Y.; Gómez, I. RSC Adv. 2014, 4, 45354.

(15) McCafferty, E.; Wightman, J. P. Surf. Interface Anal. 1998, 26, 549.

(16) Gleeson, O.; Davies, G.-L.; Peschiulli, A.; Tekoriute, R.; Gun'ko, Y. K.; Connon, S. J. Org. Biomol. Chem. 2011, 9, 7929.

(17) For selected examples, see: (a) Kainz, Q. M.; Linhardt, R.; Grass, R. N.; Vilé, G.; Pérez-Ramírez, J.; Stark, W. J.; Reiser, O. Adv. Funct. Mater. 2014, 24, 2020; (b) Linhardt, R.; Kainz, Q. M.; Grass, R. N.; Stark, W. J.; Reiser, O. RSC Adv. 2014, 4, 8541; (c) Kainz, Q. M.; Zeltner, M.; Rossier, R.; Stark, W. J.; Reiser, O. Chem. Eur. F. 2013, 19, 10038; for a review, see: (d) Kainz, Q. M; Reiser, O. Acc. Chem. Res. 2014, 47, 667. 